Usage-Based versus Measure-Based Unit Pricing: Is There a Better Index of Value?

Robert J. Kwortnik, Jr.,

Elizabeth H. Creyer and William T. Ross, Jr.

Published in the Journal of Consumer Policy (2006) 29 (March), pp. 37-66.

Robert J. Kwortnik, Jr. is an Assistant Professor of Services Marketing at the School of Hotel Administration, Cornell University. Elizabeth H. Creyer is Professor of Marketing and Logistics, Sam M. Walton College of Business Administration, University of Arkansas. William T. Ross, Jr. is Professor of Marketing at the Smeal College of Business Administration, Pennsylvania State University. 
Usage-Based versus Measure-Based Unit Pricing: Is There a Better Index of Value?

Contact Information: Elizabeth H. Creyer

302 Business Building

Department of Marketing and Logistics

University of Arkansas

Fayetteville, AR 72701

email: bcreyer@walton.uark.edu

phone:479-575-3227 


\title{
Usage-Based versus Measure-Based Unit Pricing: Is There a Better Index of Value?
}

\begin{abstract}
In many product categories, unit prices facilitate price comparisons across brands and package sizes; this enables consumers to identify those products that provide the greatest value. However in other product categories, unit prices may be confusing. This is because there are two types of unit pricing, measure-based and usage-based. Measure-based unit prices are what the name implies; price is expressed in cents or dollars per unit of measure (e.g. ounce). Usage-based unit prices, on the other hand, are expressed in terms of cents or dollars per use (e.g., wash load or serving). The results of this study show that in two different product categories (i.e., laundry detergent and dry breakfast cereal), measure-based unit prices reduced consumers' ability to identify higher value products, but when a usage-based unit price was provided, their ability to identify product value was increased. When provided with both a measure-based and a usagebased unit price, respondents did not perform as well as when they were provided only a usagebased unit price, additional evidence that the measure-based unit price hindered consumers' comparisons. Finally, the presence of two potential moderators, education about the meaning of the two measures and having to rank order the options in the choice set in terms of value before choosing, did not eliminate these effects.
\end{abstract}




\section{Usage-Based versus Measure-Based Unit Pricing: Is There a Better Index of Value?}

Unit pricing is supposed to help consumers make more informed choices. The display of prices on a per-unit weight or volume basis, the standard unit pricing scheme, should make price comparisons easier, thereby helping shoppers identify products that offer the greatest value across different package sizes within a product category (Manning, Sprott, \& Miyazaki, 1998; Russo, 1977). Research following the introduction of unit pricing in the 1970s generally confirmed that there was a cost-saving benefit to consumers who used unit prices, especially when deciding between different package sizes (Granger \& Billson, 1972; Isakson \& Maurizi, 1973; Russo, Kreiser, \& Miyashita, 1975; Russo, 1977). For example, Russo (1977) found that consumers provided with unit prices on separate shelf tags spent $1 \%$ less ( $3 \%$ less if the unit prices were in list form) than consumers without unit price information. Similarly, Zeithaml (1982) found that consumers made more economical purchases (paying an average of 8.7\% less) when unit prices were available and presented in list form. In a more recent study, Miyazaki, Sprott, and Manning (2000) reported that when unit prices were prominently presented in pricing displays, consumers spent less for the same quantity of goods relative to when unit prices were less prominently displayed. Unit pricing, at least conceptually, appears to benefit the valueconscious consumer.

Research has shown, however, that unit pricing may not be beneficial in all cases for all consumers (Aaker \& Ford, 1983). Price identification, calculation, and comparison involve multiple cognitive processes that some consumers may be unwilling or unable to perform (Manning, Sprott, \& Miyazaki, 1998; Monroe \& Lee, 1999). Mitchell, Lennard and McGoldrick (2003) cautioned that some consumers may not fully understand how unit pricing can help in comparing products. Research shows that some consumers, in particular those with low income and education levels, have difficulty comprehending unit pricing and tend to underutilize this metric (Isakson \& Maurizi, 1973; McElroy \& Aaker, 1979). Thus, some consumer segments may not benefit from the provision of unit prices.

We propose that there are product categories for which unit prices may actually confuse consumers. We further suggest that this is most likely to occur when usage rate, not unit weight or volume, better determines product value. This situation is increasingly common due to the proliferation of new products and formulations. For example, products within a number of food categories, such as cereals, snack foods, and dietary supplements, are available in different serving sizes and portions. Some may be priced based on weight while others may be priced based on volume. This is an important issue for consumers with health issues such as obesity or diabetes, and those consumers working to monitor their macronutrient (e.g., calories) or micronutrient (e.g., iron) intakes. A better understanding of the actual costs per serving would help consumers make more informed consumption choices. There are other product categories, such as herbicides and pesticides commonly found at hardware and home-and-garden stores, which are also characterized by inherent differences in quality or usage rate.

Specifically consider the situation in the laundry detergent category, one of several product categories where a measure-based unit price may be problematic. A 104-ounce package of a regular-formula laundry detergent might sell for \$4.49. A 92-ounce “ultra” formula detergent might sell for $\$ 9.39$. Which product is the better value? Based on a measure-based unit price, the regular detergent costs $\$ 0.70$ per pound, whereas the concentrated detergent costs $\$ 1.63$ per pound; this seems to suggest that the regular detergent provides greater value. However, the concentrated formula cleans 42 wash loads, whereas the regular formula cleans 
only 13. At 22 cents per wash load (\$9.39/42 washes), the concentrate is more economical than the regular formula, which costs 35 cents per wash (\$4.49/13 washes).

The example above raises an issue with significant implications for public policy makers. Is measure-based unit pricing confusing or even misleading in some product categories? If so, then a different type of unit pricing scheme is needed, one based on the rate at which the product is used. We identify this type of unit pricing as usage-based unit pricing. Laundry detergent is not the only category where consumer confusion may occur. A stroll through the grocery aisles reveals that the determination of product value in many categories can be a complex and confusing task. For example, measure-based unit pricing is likely to cause confusion in some over-the-counter drug categories because products within the same category are often sold in a variety of forms (e.g., liquid, capsule, spray) and concentrations (e.g., 50mg, 100mg, 250mg). Beverages such as Hi-C, orange juice, and Gatorade are also sold in different forms and concentrations. Paper goods such as toilet tissues and paper towels differ in thickness and absorbency levels. Hot sauces possess different levels of spiciness such that a mere drop of one brand of sauce far exceeds the fire power of a larger serving of another brand. Herbal remedies and nutritional supplements appear in all manner of form and potencies. Alcoholic beverages come in an array of proofs (alcohol by volume) both across and within categories (e.g., distilled liquors, beer, and wine); for instance, an ounce of whiskey has 10 times the alcohol of an ounce of beer, and some beer brands pack two to three times the punch as the typical light beer. In sum, products of many types are sold in varying concentrations that may confuse consumers because measure-based unit price is an inappropriate metric for determining the best value.

The purpose of this paper is to provide researchers, marketers, and public policy makers with insight into potential difficulties faced by value-conscious shoppers. The first objective is to determine whether measure-based unit pricing used in some product categories confuses consumers. The second objective is to examine whether the availability of usage-based unit price increases the attractiveness of higher-value products in some product categories. The third objective is to examine whether the problems associated with a measure-based pricing scheme persist in the presence of possible mitigating factors such as information-processing differences. In the next section, we provide a brief overview of this controversial issue. Following that, we develop our research hypotheses and then describe two studies that test the impact of different combinations of measure-based and usage-based unit price indices on consumers' assessments of product value. Finally, we discuss the public policy and marketing implications of the findings.

\section{RESEARCH ISSUE}

Before the introduction of unit pricing, Russo (1975) referred to pricing displays as an “information-processing nightmare.” The Weights and Measures Division is one of the longestrunning and best-known programs of the National Institute of Standards and Technology (NIST). Its primary goal is to promote uniformity in U.S. weights and measures laws, regulations, and standards to achieve equity between buyers and sellers in the marketplace. The Office of Weights and Measures established the National Conference on Weights and Measures (NCWM), an organization comprised of representatives from state and local governments, industry, consumer groups, and Federal agencies. Although the NCWM established standard pricing practices, these do not become law until adopted by state or local governments; only 20 states within the United States have adopted recommended unit pricing regulations. However, many 
larger retail chains provide unit pricing information on a voluntary basis. In sum, grocery stores are still, for some consumers, an information processing nightmare.

For example, until the early 1990s, manufacturers of laundry detergents developed the same form (powder or liquid) to be used at the same rate per wash load across brands (e.g., one cup or 8 oz. per normal load). Under these circumstances, a measure-based unit price was a convenient and useful value metric. However, "ultra” versions of popular brands were introduced and sold with varying strengths, such as 4/10-cup (3.2 oz. per load) and "quarter-cup" concentrates (some use $2 \mathrm{oz}$. per load, others $2.2 \mathrm{oz}$.). This weight-per-load index is not printed on packages; the only way to ascertain the actual strength is by dividing the total package weight by the wash-load count stated on some, but not all, package labels. Odd-lot total weights used by manufacturers compound the complexity. For instance, one brand has a 40-wash load product with a package weight of 8.37 pounds. To arrive at a strength estimate, one must: 1) convert pounds to ounces (8.37 lbs. x $16 \mathrm{oz} .=134 \mathrm{oz}$.$) ; 2) divide total ounces by total wash loads (134$ oz. $\div 40$ wash loads $=3.35$ oz./wash load); 3 ) divide this ratio by the number of ounces in a traditional cup (3.35 oz./wash load $\div 8=.42$ ); and finally, 4 ) make an approximate judgment.

Even if consumers can estimate usage-rate information for a specific product, making comparisons across different products within a category can be difficult. The number of stockkeeping units (SKUs) offered by manufacturers has proliferated to such an extent that substantial differences in package weight and size, both within and across brands, are now the rule rather than the exception. For example, a Consumer Reports (1995) test of ultra formula detergents included 14 different package sizes; the volume of the packages ranged from 32 to 135 ounces. Not surprisingly, manufacturers typically offer products across a range of sizes that differ from the sizes offered by competitors, presumably to prevent direct consumer comparisons. Choice complexity further increases when varying formula strengths (e.g., dilution required versus dilution not required) and differing product forms (liquid versus powder) are added to the shopping environment.

Consider another category, breakfast cereals. An examination of the smallest multiserving package size for five different brands indicated the following: package weight varied between 15 ounces and 18 ounces, a relatively small variation, but still sufficient to force deliberation if one was to calculate value. Perhaps more importantly, servings per package varied from 8 to 11, and the larger number was not associated with the larger package weight. A growing proportion of U.S. consumers are overweight and/or developing adult onset diabetes, predominantly because they are overweight, which has led to a focus on serving size and portion control. Yet this complexity in one of the simpler and ubiquitous food categories may make serving-size management difficult; this reveals a significant issue for public policy makers.

Consumer information-processing difficulties in the shopping environment may also have serious negative implications for marketers. Sales evidence suggests that consumers are indeed confused. For example, consumers were slow to adopt concentrated formulations of popular laundry detergent brands because of the mistaken perception that they would pay more while receiving less (Marketing News, 1995). Despite the better value and reduced-packaging benefits associated with ultra detergents, regular-strength products continued to be strong sellers years after the introduction of same-brand concentrates and were even outselling ultra formulas in some markets (Turcsik, 1994; Walsh \& Hume, 1999, 2000). Moreover, some consumers who used concentrated formulas did not seem to believe that "less" of an ultra product could clean as well as the traditional amount used with regular-strength products. Category managers noted that the switch to ultra detergents was followed by an unexpected increase in unit sales (Turcsik, 
1994). This suggested that consumers were overusing the product, either out of habit or confusion, by using the same amount, or perhaps only a small amount less, of ultra detergent per wash as would be used with regular-strength detergents (Bauer, 1994). Despite this evidence, empirical research to date has not provided an answer to an important question: do consumers make poorer choices because of measure-based unit pricing?

In sum, different package sizes, varied formulations, different serving sizes, and potentially confusing pricing displays in many product categories may contribute to poor consumer choices. Short of requiring standardizing product formulas and packaging, a solution for this problem is the implementation of a more diagnostic value metric, one that enables meaningful product comparisons across package sizes and product concentrations. Consistent with the recommendations of consumer advocates (Breskin, 1995; Marketing News, 1995), we propose that a usage-based unit price is a more informative cue than measure-based unit price for those product categories that that feature alternatives with different usage rates.

\section{HYPOTHESIS DEVELOPMENT}

\section{Measure-based Unit Pricing}

Consumers face a complex information environment in many product categories. Research suggests that one way consumers cope with task complexity is by utilizing less effortful information integration strategies and by employing simple decision heuristics (Bettman, Johnson, \& Payne, 1991; Johnson \& Payne, 1985). For example, shoppers may use an elimination-by-aspects strategy (e.g., "choose the most convenient package size, then choose the lowest priced brand in this size”) or a lexicographic strategy (e.g., choose the brand with the lowest price). In fact, Hoyer (1984) found that nearly three out of four detergent buyers only looked at one package and less than one in 20 examined more than two options. One of the consequences of using a heuristic that minimizes effort is that non-diagnostic information may be weighted too heavily in the decision process.

Slovic (1972) found support for a “concreteness” principle; people tend to use information in the form in which it is presented rather than expend the cognitive effort to transform it. Russo's (1977) finding that consumers made better decisions when unit-price information was provided in list form rather than by individual shelf tags also underscored the importance of information format. When comparing brands across product forms, such as powder laundry detergents and liquid laundry detergents, consumers must gather information from a) the shelf tag for overall price and unit price and b) the product package for use or serving and package weight information. It is doubtful that most consumers have the ability or motivation to integrate this assortment of information.

Given the range of prices and package sizes in many categories in the typical supermarket, consumers are likely to use the pricing information that is the easiest to process. In certain product categories, measure-based unit pricing provides diagnostic information that enables consumers to choose the product that offers the best value (Granger \& Billson, 1972; Russo, 1977). However, the presence of products with different formulations or serving sizes complicates the preference formation process. For example, when the choice set is mixed, that is, when concentrated formulas are presented alongside "regular-strength" products, a measurebased unit price is not diagnostic. Products with greater concentrated strength often will be more expensive per unit weight, volume, or count than regular formulas, even if the concentrates are a 
better value. This suggests that measure-based unit prices may hinder the consumer's ability to determine value. Thus, our first hypothesis is that:

H1: Measure-based unit prices will hinder the consumer's ability to choose higher value products in categories that feature alternatives with different usage rates.

\section{Usage-based Unit Pricing}

For many product categories, when consumers try to make an informed choice based on product value, the strength of the product formula or the serving size must be considered. For instance, in the laundry detergent category, wash-load count is sometimes, but not always, presented on the package and serves as a proxy measure of strength. Number of wash loads may appear on packaging either in words (e.g., "Large Size 40 Loads”) or as a number inside a scoop or cup illustration. Such descriptions provide consumers with some measure of usage rate that can be integrated with package size and price information to approximate a price-per-wash value estimate. Note that some non-concentrated brands (often ironically called "value" brands) do not provide consumers with any information regarding the number of loads washed per package.

We propose that information regarding the number of uses per package, which is comparable to the number of servings per container, serves as a more diagnostic indicator of product value than measure-based unit price because consumers can apply the number of uses to estimate a price/usage index for themselves. Consumers who integrate the number of uses per container (e.g., wash loads) and total price information should better discriminate value levels between brands with different formulas and prices. However, while number of uses is useful information, we argue that the most useful information is not generally available - price per use. Creyer and Ross (1997) have demonstrated that a value index serves as a diagnostic cue, influencing consumer decision making towards higher value products. The price-per-use index, the exemplar of a usage-based unit price, should have diagnosticity similar to or greater than that of the number of uses per package. However, the price-per-use index should be easier to process because it integrates both price and use (e.g., wash load) information in a single metric. Only this cue is needed to assess product value; additional mathematical calculations are unnecessary. Because price per use is a cognitively simpler metric to use, it should be superior to the numberof-uses-per-package count.

H2: Consumers provided a usage-based unit price will be better at choosing higher value products in product categories that feature alternatives with different usage rates.

\section{Measure-based and Usage-based Unit Pricing}

An important question is whether pricing displays that feature measure-based unit price information and also a number-of-uses count and/or a price-per-use index will increase confusion and thus decrease the accuracy of the value assessments or whether they will act as redundant information. As we have suggested, measure-based unit price may provide a false measure of value in product categories that feature alternatives with different usage rates. Whether the presence of both measure-based unit price and the usage-based indices has a negative impact on the consumer's ability to accurately identify product value will depend on the specific type of information used by the consumer. Bettman et al. (1991) reported that choice accuracy typically improved with the addition of attribute information. However, they also noted that as 
information load increased, decision quality sometimes decreased. The authors concluded that consumer accuracy in decision-making was contingent upon task variables as well as context effects due to the nature of the information available to - and used by - the decision maker.

On the other hand, Bettman et al.’s (1991) finding was not concerned with information that may actually conflict and create confusion. The presence of mixed-usage products in a category is a context effect that is expected to reduce choice accuracy, unless there is simple, diagnostic information available at the point of purchase to aid decision-making (Johnson \& Payne, 1985). For example, in a mixed-usage product set, when consumers are shown overall prices of alternatives, measure-based unit prices, and the number of uses per package or a priceper-use index, choice based only on overall price will not be optimal. To make an accurate value assessment, it is necessary to integrate additional information. If consumers compare total price and the number of uses per package across brands, judgments should be relatively accurate. Value accuracy should also be attained if consumers compare price per use across brands. However, based on their experience in other categories, some consumers are also likely to use the measure-based unit price (Aaker \& Ford, 1983). If both types of information (measure-based and usage-based) are given equal weight during preference formation, the consumer's ability to form an accurate value judgment will be diminished. Thus, the presence of measure-based unit price should inhibit the processing of the more diagnostic usage-based information.

H3: Consumers faced with both measure-based and usage-based information in product categories that feature alternatives with different usage rates will not choose the higher value product as often as will consumers who are provided with only the usage-based unit price information.

\section{STUDY 1}

Study 1 was designed as a direct test of the hypotheses presented above. Participants had the task of choosing one product from two choice sets, one from the powdered laundry product category and a second from the dry breakfast cereal product category. Products were described in terms of price and weight and some form of unit pricing information. The nature of the unit pricing information participants had to assist them in their decision was one four-level factor in the study design. To test for moderating effects, two additional factors were manipulated, whether or not the participants were provided with an explanation of the unit price information they received and whether or not participants were asked to rank order the alternatives in terms of value before they made their choices.

\section{Participants, Procedure, and Instrument}

Participants were 216 students who took part as partial fulfillment of requirements for a marketing course at a large South-Central University. Questionnaire administration was by paper and pencil at the end of a normal course session. Data from 7 participants were deleted because of missing information, leaving a final sample of 216. Table 1 contains the two choice sets.

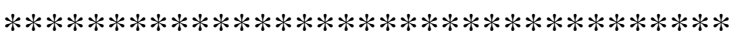

Insert Table 1 about here 


\section{Design}

The design was a three-factor between subjects design. The first factor was the nature of information that respondents had about the alternatives in the choice set and had four levels: 1 ) measure-based unit price only, 2) usage-based unit price only, 3) both measure- and usage-based unit price, and 4) neither measure-based nor usage-based unit price information (recall that participants had package weight and package price for all items). The second factor had two levels: whether or not the participants were provided with an explanation of the unit price information they received. The third factor also had two levels: whether or not participants were asked to rank order the alternatives in terms of value before they provided their choices. Thus, the design was a $4 \times 2 \times 2$ between subjects design.

\section{Results and Discussion}

Separate analyses were run for the laundry detergent and breakfast cereal categories. For each category, two analyses were run, one to examine how choice patterns changed for the bestvalue product based on a usage-based unit price metric and the other to examine how choice patterns changed for the best-value product based on a measure-based unit price metric. Implicit in this analysis is an important assumption, that is, that the product with the lowest usage-based unit price is the best product. This assumption is necessary in order to examine whether or not the presence of a usage-based unit price increases the consumer's likelihood of choosing highervalue products. In each case, a logistic regression was run with whether or not the product in question was chosen as the 0/1 dependent variable and the design levels for the three factors as the independent variables. Results are displayed in Table 2. We discuss each below.

$* * * * * * * * * * * * * * * * * * * * * * * * * * * * * * * * * * *$

Insert Table 2 about here

For laundry detergent B, the lowest-cost alternative based on price per ounce and the second highest cost based on price per use, two variables predicted the degree to which it was chosen. When price per use was included, the likelihood of Brand B being chosen decreased significantly $(\mathrm{B}=-.929, \mathrm{p}<.03$ ), and when price per ounce was included, the likelihood of it being chosen increased marginally $(\mathrm{B}=.675, \mathrm{p}<.09)$. Neither the provision of explanations about the unit pricing information nor instructions to value rank alternatives had any effect. For laundry detergent $\mathrm{D}$, the lowest-cost alternative based on price per use and the third highest cost based on price per ounce, one variable predicted the degree to which it was chosen. When price per ounce was included, the likelihood of $\mathrm{D}$ being chosen decreased significantly $(\mathrm{B}=-.637, \mathrm{p}<$ .04). Neither the provision of explanations about the unit pricing information nor instructions to value rank alternatives had any effect.

For breakfast cereal D, the lowest-cost alternative based on price per ounce and the fourth highest cost based on price per serving, two variables predicted the degree to which it was chosen. When price per ounce was included, the likelihood of it being chosen increased significantly $(\mathrm{B}=.726, \mathrm{p}<.05)$, and when value rankings were elicited, the likelihood of it being chosen decreased marginally significantly $(B=-.589, \mathrm{p}<.09)$. For breakfast cereal $\mathrm{C}$, the lowest 
cost alternative based on price per serving and tied for second highest cost based on price per ounce, two variables predicted the degree to which it was chosen. When price per ounce was included, the likelihood of it being chosen decreased significantly $(\mathrm{B}=-.849, \mathrm{p}<.01)$, and when descriptions of the unit pricing schemes were provided, the likelihood of it being chosen increased marginally significantly $(\mathrm{B}=.542, \mathrm{p}<.08)$.

How do these results relate to our hypotheses? When price per ounce is statistically significant, it reduces choice for the best product based on usage and increases choice for the best product based on measure. Similarly, when price per use is significant, it reduces choice for the best product based on ounces and increases choice for the best product based on usage. Given the assumption that basing value on price per use is better, this evidence supports both Hypothesis 1, that measure-based unit prices hinder the consumer's ability to choose highervalue products in categories that feature alternatives with different usage rates, and Hypothesis 2, that consumers provided with a usage-based unit price will be better at choosing higher value products. Hypothesis 3 is supported by choice results for laundry detergent $\mathrm{B}$, the best product given price per ounce but not given price per use. That is, consumers faced with both measurebased and usage-based information would not choose the higher value product as often as would consumers who are provided with only the usage-based unit price information.

The results of this study show that, for two different product categories, the provision of typical measure-based unit price information hindered the consumer's ability to choose highervalue products. That is, price per ounce led consumers to increase their purchase of the best value product based on unit price and decrease their purchase of the best product based on price per use. These results support our contention that shoppers still face information-processing challenges when choosing among alternatives in product categories that feature alternatives with different usage rates. There are limitations of Study 1 which reduce the generalizability of our results to a wider range of both consumers and purchase situations. Thus, Study 2 was conducted with both more realistic stimuli and an adult, nonstudent sample. While there was no reason to expect our results not to generalize, we considered confirmation useful, given the somewhat controversial nature of our results.

\section{STUDY 2}

\section{Participants}

Participants from a consumer panel $(\mathrm{N}=402)$ participated in the study by mail. Their task was to rate the attractiveness of four fictitious brands of powder laundry detergent that varied in terms of formula concentration. The brands were represented by mockups of product packages and shelf tags shown on a four-panel display on one page of the experimental booklet. Each subject was presented three choice sets (one set per page) of four brands each with the following characteristics. Set 1 contained similar package sizes. Set 2 presented products with similar overall prices. Set 3 showed products with equivalent use-per-package counts. Although these variations altered the total price and package size of each brand across the sets, the measure-based unit price and price-per-use index of each brand remained constant across the sets. Finally, the presence of measure-based unit price, number-of-uses-per-package, and priceper-use information varied between subjects across eight conditions.

\section{Stimuli}


The stimuli were designed to simulate the information environment typically encountered by consumers, while still controlling for brand name and package-size influences. To control for brand name effects, four fictitious brands were developed based on real brands. The most attractive brand in each set, based on a computed price-per-use index, was modeled after the Arm \& Hammer 4/10-cup ultra detergent. The second most attractive brand was based on the Bold quarter-cup ultra detergent. The third most attractive brand was based on the Tide quartercup ultra detergent. Finally, the least attractive brand in each set was modeled after the Purexbrand one-cup "classic" formula detergent. Together these alternatives represent a good crosssection of the brands in their category, different concentrations, and different pricing strategies.

The brands were presented such that their value was ordered randomly (see Table 3 for a summary of the information used to create the stimuli profiles). Similar to an actual retail environment, price, volume, and usage information was displayed either on the product packaging or on a simulated shelf tag that appeared below the package illustration. The layout of this information was consistent across brands and sets.

Insert Table 3 about here

The three sets were designed to control for the fact that consumers can be expected to approach brand comparison tasks differently. To control for any effect of package-size, package sizes were kept similar in Set 1 . Package weights are only marginally different and package sizes were modified slightly to reflect this minor weight differential. To achieve size comparability across brands, total prices and number of uses per package were rescaled based on actual product information. Brands in this set were labeled A, B, C, and D.

Set 2 (similar price) controls for total price as a primary input to brand comparisons. The total prices in this set were similar, ranging from $\$ 4.29$ to $\$ 4.59$. To account for differences in formula concentration, package sizes and number of uses per package were rescaled. The logic behind Set 2 is that some consumers might employ a heuristic that first groups alternatives in terms of similar prices and then forms preferences based on other factors. The same base stimuli were used from Set 1 to develop Set 2, though the package design was altered to minimize carryover effects and brand names were changed to Brands E through $\mathrm{H}$.

Set 3 (same number-of-uses-per-package) controls for the wash-load cue that appears on many detergent packages. Each alternative featured the same 42-wash-load count, and total price and package size were rescaled. The logic behind Set 3 is that some consumers might employ a heuristic where alternatives are first grouped by equivalent total usage. An interesting aspect to this set is that by equalizing the wash load count, a measure of use is built in. That is, consumers should be able to identify the best-value product simply by ranking them according to price because each product cleans the same number of laundry loads. However, package size might confound this determination because of differences in formula concentration. For instance, the regular formula (one-cup) brand in this set has roughly three times the volume of the concentrated brands. On the other hand, the total price of this brand is the highest in the set while 
being the lowest in the first two sets. Again, the brand name design was altered, and names were changed to Brands I through L.

Subjects were instructed to rate each brand on a 1 to 7 scale based on how attractive the brand would be if they were purchasing laundry detergent, with 1 being "not at all attractive" and 7 being "very attractive." The sets were constructed such that there was a "true" order of attractiveness based on product value. Once again, we assume this "true" order of attractiveness because our purpose is to examine how well consumers recognize the best-value brand in terms of its usage rate. However, using a rating scale rather than a rank order measure allowed for the possibility that some subjects might be indifferent between brand alternatives and unable (or unmotivated) to rank order the brands. Consumers were asked to provide ratings of brand attractiveness, rather than ratings of product value, because we felt that this task better replicates actual consumer-shopping behavior.

\section{Design}

The study used a $2 \times 2 \times 2 \times 3$ full-factorial design, with the presence or absence of three independent variables, measure-based unit price, number of uses per package, and the price-peruse index, as between-subjects factors and choice set as a within-subjects factor. Subjects were randomly assigned to one of eight conditions, with approximately 50 subjects per condition. Subjects in all conditions saw package weight and total price information on the shelf tag. For subjects in Condition 1, this was the only information available to assess product attractiveness. Subjects in Conditions 2 through 8 saw some combination of the independent variables, with subjects in Condition 8 viewing all three indices.

\section{Results and Discussion}

To determine the quality of consumers' assessments of product value across conditions and processing sets, we performed the following analysis (Cohen \& Cohen, 1983). For each subject and for each choice set within subject, we calculated the correlation between the rank order of the subject's attractiveness ratings and the "true" ordinal ranking of the brands based on price per wash. This correlation was converted to a z-score. This z-score served as the dependent measure for an ANOVA based on the $2 \times 2 \times 2 \times 3$ design. Smaller z-scores indicate less accurate value assessments. This analysis is done because our interest is in the degree of overall conformation to an ordering that emphasizes greater value, not in how evaluations change across brands or sets based on our manipulations. Thus, it is the structure of the set of responses that is important not the response to any individual brand, so it is the correlation between the respondent's evaluations and the "true" ordinal rankings based on price per wash that is necessary for this analysis.

Insert Tables 4 and 5 about here 
Table 4 presents the ANOVA results, and Table 5 presents the average $\mathrm{z}$-scores for each condition. Recall that the choice set manipulation was used to control for differences in how consumers might process the information. As the first panel of Table 4 indicates, there was a statistically significant $\left(\mathrm{F}_{2,338}=23.117, \mathrm{p}<.001\right)$ main effect for choice set, but none of the interactions between choice set and the other manipulations was statistically significant. With respect to the main effect for choice set (Table 5), the mean z-score for the first set (composed of approximately the same size packages) is lower $(\mathrm{M}=0.766)$, indicating a lower correlation and hence a lower performance, than the mean z-score of the second set $(\mathrm{M}=0.991)$ which was composed of products with approximately equivalent prices. This, in turn, is lower than the mean z-score of the third set $(M=1.115)$, which was composed of products that offered the same number of uses (i.e., wash load counts). The 95\% confidence interval for the mean of the first set does not overlap with the same interval for the mean of the third set, and the overlap between the first and second sets is almost but not quite nil. These results suggest that, as expected, having an equivalent number of uses in the choice set, as in set 3 , simplified the task the most, and that equivalent price, as in set 2 , was next in simplicity. The non-significance of the interactions between choice set and the other manipulations indicates that the results are reliable across the different situations faced by the respondents. Thus, these interactions are discussed no further. In the following section we examine the results pertaining to the study's hypotheses.

We proposed that measure-based unit price is not a good cue in product categories that feature alternatives with different usage rates and, therefore, will not improve, and may even harm, consumers' value assessments. Table 4 shows that the presence of measure-based unit price does not improve value assessment $\left(\mathrm{F}_{1,338}=.530\right.$, NS). Examination of the means (Table 5) indicates that the $\mathrm{z}$-score is lower when measure-based unit price is present $(\mathrm{M}=.920)$ than when it is not present $(M=.995)$, although the difference is not significant. This indicates that measure-based unit price does not improve performance, although its harmful effect, as evidenced by the reduction in the overall mean for those conditions in which measure-based unit price is present from those in which it is not present, is not sufficient to achieve statistical significance. However, an additional test of this proposition is to determine the effect of measure-based unit price on performance, absent all other manipulations. To do this, we perform a simple contrast of the condition in which no unit pricing information is provided with the condition in which only measure-based unit pricing is provided. For each of the three sets, the difference is always in the same direction. For set 1 , the presence of unit price results in a mean $\mathrm{z}$-score reduction of $0.1372\left(\mathrm{t}_{105}=1.387, \mathrm{p}=.084\right.$, one-tailed); for set 2 the mean $\mathrm{z}$-score reduction is $0.2115\left(\mathrm{t}_{103}=1.543, \mathrm{p}=.063\right.$, one-tailed); for set 3 the mean $\mathrm{z}$-score reduction is $0.0967\left(\mathrm{t}_{102}=0.537, \mathrm{p}=.296\right.$, one-tailed). Across the three sets, the averaged mean $\mathrm{z}$-score difference is significant ( $\mathrm{t}_{314}=1.755, \mathrm{p}<.05$, one-tailed). Another way to analyze this data is with the Chi-Square statistic. Specifically, two times the log likelihood of a probability is a ChiSquare with one degree of freedom. The sum of Chi-Squares is also a Chi-Square with the sum of the degrees of freedom of the constituent Chi-Square statistics. Thus, the overall probability of the summed Chi-Square can be assessed, which provides an independent assessment of the total effect of the measure-based unit price across the three choice sets. When this test is performed, the Chi-Square statistic that represents the three sets is 12.918, with 3 degrees of freedom, which is statistically significant at the $\mathrm{p}<.001$ level. The results are clear; measure-based unit pricing does not improve, and when other unit price information is absent, harms value assessment in product-choice sets that feature alternatives with different usage rates. 
The presence of the number-of-uses-per-package measure was expected to improve consumers' value assessments. Table 4 shows this to be the case $\left(\mathrm{F}_{1,338}=18.443, \mathrm{p}<.001\right)$. Examination of the means indicates that the mean z-score is significantly higher when the number-of-uses-per- package count is presented $(\mathrm{M}=1.180)$ compared to when it is not presented $(\mathrm{M}=0.735)$. Thus, the presence of number of uses per package does improve value assessment as predicted in product-choice sets that feature alternatives with different usage rates.

We also proposed that the presence of a usage-based unit price will improve the accuracy of consumer value judgments and to a greater extent than will the presence of the similar but more difficult to process number-of-uses-per-package measure. Table 4 shows that the presence of usage-based unit price does improve value assessment $\left(\mathrm{F}_{1,338}=61.457, \mathrm{p}<.001\right)$.

Examination of the means indicates that the mean z-score is significantly higher when usagebased unit price is present $(\mathrm{M}=1.363)$ than when it is not present $(\mathrm{M}=0.551)$. To examine whether a usage-based unit price is more effective than the number-of-uses measure, we use the means from the usage-based-unit-price by number-of-uses (i.e., wash load count) interaction to compare the condition in which a usage-based unit price and not number of uses is present $(\mathrm{M}=$ 1.331) and the condition in which number of uses is present and not usage-based unit price $(\mathrm{M}=$ 0.964). There is minimal overlap between the 95\% confidence intervals for these two conditions indicating that the two means are most probably different from each other. Thus, as expected, the presence of usage-based unit price does improve value assessment, both in the absolute and compared to including the number-of-uses index in mixed-usage product-choice sets.

The combined effect of usage-based unit price and the number-of-uses index was expected to be smaller in magnitude than the sum of the main effects for these indices due to information redundancy. Table 2 shows that the interaction is statistically significant $\left(\mathrm{F}_{1,338}=\right.$ 13.458, $\mathrm{p}<.001)$. Examination of the means indicates that the mean for the condition in which number of uses is included with usage-based unit price $(M=1.396)$ is not significantly different from the mean for the condition in which the usage-based unit price alone is present $(M=1.331)$, although the condition in which both are present (again $M=1.396$ ) is significantly different from the condition in which only number of uses is present $(M=.964)$. Here there is no overlap between the two conditions' 95\% confidence intervals. Thus, we see that provision of number of uses does not provide additional information to provision of a usage-based unit price.

We also proposed that the inclusion of measure-based unit price information would attenuate the effects of both the usage-based unit price and the number-of-uses information. Table 4 indicates that neither of the two-way interactions that include measure based unit price, measure-based unit price by number of uses $\left(\mathrm{F}_{1,338}=0.083, \mathrm{NS}\right)$ and measure-based unit price by usage-based unit price $\left(\mathrm{F}_{1,338}=0.623\right.$, NS) is statistically significant. In addition, the three way interaction $\left(\mathrm{F}_{1,338}=2.753, \mathrm{p}=.084\right)$ is only marginally significant. Together these results show a clear pattern. The presence of measure-based unit price does not significantly attenuate the effects of other cues, although the means for all three interactions are in the hypothesized direction. This interesting result suggests that the presence of usage-based unit pricing is sufficiently helpful as a value cue such that measure-based unit pricing cannot override it enough to reduce the quality of the evaluations.

\section{GENERAL DISCUSSION}

Much has changed in the supermarket-shopping environment in the 30 years since researchers first examined the influence of unit pricing on consumer decision making. One 
significant change has been the introduction of concentrated formulas in a number of different product categories. The results of our studies provide evidence that the traditional measure-based unit price does not offer the benefit of easier and more accurate across-brand value comparisons in product categories such as laundry detergent and breakfast cereals, the two categories we studied, and presumably many others that contain product alternatives used at different rates. A goal of unit pricing is to help consumers make more economical purchase decisions. However, our research signals the need for changes to current public policy regarding this goal.

Using two manipulated judgment tasks, one with students and one with a consumer panel, we find that people have difficulty accurately assessing product value in product-choice sets that feature alternatives with different usage rates using only total price and measure-based unit price information. We expected that the use of the measure-based unit price cue might impair consumer judgment; this was shown in the first study but not in the second. In both studies, consumers tended to be less accurate when measure-based unit prices were present in pricing displays, which suggests some degree of consumer confusion. However, this inaccuracy did not significantly harm value assessment. In addition, measure-based unit prices did not significantly improve preference quality. Thus, we can conclude that measure-based unit price is not a helpful value metric for making choices within product categories that contain alternatives with different usage rates or product strengths.

On the other hand, our results show that usage-based indices are diagnostic and do facilitate across-brand value comparisons. Both the presence of the number-of-uses-per-package cue and a usage-based unit price in pricing displays significantly improved consumer value assessments. The usage-based unit price proved to be the most diagnostic, and consumers were not more accurate when both the number-of-uses-per-package and usage-based unit price indices were present, at least compared to the usage-based unit price metric alone. Measure-based unit price did not significantly harm value assessment when consumers had either or both usage indices available. Taken together these results suggest that the simplest and most effective way to aid consumer decision-making is to augment the current measure-based unit pricing system with a usage-based unit pricing index and to educate consumers about the new metric.

Indeed, the value of usage-based labeling has been recognized by the U.S. Food and Drug Administration (FDA). They note that serving size is especially critical to nutrition labeling since all of the information on nutrient levels depends on the amount of the product represented. However, serving size, which is currently required by the Nutrition Labeling Education Act (NLEA) to be present on the nutrition facts panels of most packaged food products, may differ dramatically from the amount typically consumed, that is, the usage-based amount. For example, a bag of cheese curls that is only slightly larger than a "typical" single size serving of chips and can easily be consumed at one eating occasion may contain $3 \frac{1}{2}$ servings. Thus, the FDA is currently considering whether to make changes to the NLEA that would require manufacturers to provide the nutrition information for the whole package when that package can reasonably be consumed at one eating occasion.

\section{IMPLICATIONS FOR PUBLIC POLICY}

Concentrated-formula products generally offer consumers several benefits: a better peruse value when compared to most regular-formula brands, the convenience associated with a smaller package, and the social benefits derived from choosing a product form that places less stress on the environment (i.e., fewer resources used in creating the product and its package, and 
less landfill space required for package disposal). However, such benefits may be overshadowed if consumers are confused by the explicit value information suggested by unit prices when compared to the implicit value levels offered by products with different usage rates. The result of this confusion may be a failure to choose the highest-value product because the consumer does not fully understand that a smaller amount of concentrate can provide the same performance as a larger amount of a traditional version. Although usage rates could not be examined in this paper due to the methodology used, we speculate that even when the highest-value product is purchased, it may not necessarily be used in the most optimal way.

For product categories that have brands with varied usage rates, the presence of a usagebased unit price can help to mitigate these problems. Such an index is an example of what Johnson, Payne, \& Bettman (1988, p. 19) call "passive decision support” - an information display that can produce improved decisions through “...passively [encouraging] better strategies by making them easier to execute.” Although measure-based unit pricing is such a decision aid for many product categories, the results of this study show that it did not produce better decisions for categories in which product-usage rates vary. In fact, consumers generally found the highervalue concentrated brands less attractive and the lower-value regular-formula brands more attractive when measure-based unit price was present. Just the opposite occurred when the usagebased unit price metric was introduced into the consumer environment. This positive effect of the usage-based metric is especially important when one considers the research which shows that the consumers most likely to buy the low-priced, dubiously named "value" brand, regular-strength products are the people least able to afford a poor-value choice (Isakson \& Maurizi, 1972; McElroy \& Aaker, 1979). Thus, if a usage-based value index not only leads to better decisions for most consumers, but also minimizes the chance that less vigilant consumers will be misled by pricing tactics and promotional wording on package labels, then the implementation of such an index has consumer-protection benefits as well.

It is interesting to speculate about why manufacturers of "ultra" products in categories such as laundry detergent and the retailers who sell these products have not adopted a usagebased unit pricing scheme. Such a measure should benefit the concentrate manufacturers by helping consumers to see that the concentrates had the same or better value than the nonconcentrated products, and we speculate that this is the reason that the manufacturers have included signals such as the number of wash loads on their packaging. However, pricing, and thus the usage-based unit price, is controlled by the retailer, not the manufacturer; the manufacturer cannot dictate the price at which the retailer sells the product (Garman, 1996), so, the manufacturer cannot control the usage-based unit price. Moreover, retailers are not likely to voluntarily provide a usage-based unit price, as this would be additional work for retailers who would have to maintain information about usage rates to calculate the measure and because the constant price discounting present in the retail environment would be complicated. Thus, it may be not be in the retailer's perceived best interests to provide information that would reduce the amount sold in important categories. Taken together, this suggests that if a usage-based measure is needed by consumers to make informed choices, the Weights and Measures Division of the NIST may have to take the initiative. Ideally, those states that have already adopted unit pricing regulations would be willing to introduce a usage-based pricing scheme into the marketplace if such regulations were drafted by the NIST.

\section{IMPLICATIONS FOR MARKETERS}


Consumer confusion resulting from non-diagnostic pricing information at the point of purchase may create problems for manufacturers and retailers. Quick acceptance of new products can reduce the need for costly test-marketing and promotion programs designed to stimulate product trial. Such savings would be most beneficial for brand extensions and new-product launches in product categories dominated by regular-strength products. Once consumers accept and understand the performance equality of a concentrate formula, this usage knowledge can be transferred to similar usage situations, such as "ultra" car wash detergents and household cleaning products. Furthermore, there are the cost economies gained by cutting production and distribution costs.

If the challenges experienced by laundry detergent manufacturers are any indication, perceptions of diluted value caused by the use of non-diagnostic pricing information also threaten the firm that is first to market with an innovative, new concentrated product. For example in 1976 Colgate-Palmolive introduced Fresh Start powder, a quarter-cup concentrate. Despite a first-mover advantage, Fresh Start really never caught on and held only a $2 \%$ share of the market by 1991. The problem, according to a Colgate executive, was Fresh Start's comparatively smaller package size, which fostered consumers' perceptions that they received less product for the money (Appelbaum, 1991). Proctor and Gamble experienced similar problems when introducing Tide concentrate in the Canadian market in the early 1990s and was forced to reintroduce the traditional formula. According to a P\&G spokesperson, "People thought they were buying the (bulk powder) formula for the same price but in a package half the size” (Mullin, 1992). Even after concentrates attained consumer acceptance in the powder category, Lever Brothers had to backpedal on a move to quarter-cup liquid ultras from a half-cup form after sales declines of 14 percent for Wisk and 30 percent for All. Said one manufacturer spokesperson, it appeared the industry had hit a "boundary” with consumers (Breskin, 1995).

If these problems were unique to laundry detergents, one might chalk it up to the normal product adoption curve. However, history was repeated in 1998 when P\&G abandoned its Mr. Clean and Spic 'n Span concentrates and returned to regular-strength formulas after the firm's market share in the all-purpose household cleaner category plummeted from 20.3 percent to 11.6 percent - despite four years of promotional support (Neff, 1999). In summary, consumer confusion caused by packaging differences, varied concentrate strengths, and uninformative pricing displays may have deleterious effects for marketers if possible first-mover advantage is lost because the innovative product may be seen as more expensive than regular formulas when in fact it may provide a real improvement in customer value. The implementation of an easy-tounderstand and more diagnostic usage-based unit price may reduce the risk of product failure for first-movers, especially after consumers become aware of and understand the information conveyed by the new metric.

Concentrated products also offer retailers a major benefit that is increasingly scarce and valuable - additional shelf space. Thanks to the smaller packages required of concentrates, retailers have the opportunity to increase the facings of fast-moving brands and sizes and/or to dedicate more shelf space to new products and line extensions in the product category. However, this benefit is only realized if the shift to concentrates is relatively expedient. When slow consumer adoption of new formulas hinders product turnover, this actually can strain shelf space, as retailers are forced to carry both concentrates and regular-strength products to satisfy the demand of early adopters as well as traditional buyers.

In sum, a switch to the marketing of concentrated products within a category offers gains for stakeholders at various levels in the value-delivery chain. However, these gains can be 
stunted if consumer adoption of the new products stalls due to misunderstanding of the value equation. The example of the problems encountered by laundry detergent manufacturers during the introduction of ultra products shows how measure-based unit pricing and inadequate consumer education contributes to this consumer confusion and resistance to change.

\section{LIMITATIONS}

The results of this study offer evidence that consumers make use of different pricing cues for making a best-value decision, a finding that is consistent with prior research on unit pricing (e.g., Aaker \& Ford, 1983; Miyazaki et al, 2000; Russo, 1977). However, research in the area of price perception also suggests that many consumers do not fully attend to or diligently process price information, especially for low-involvement purchases (see Dickson \& Sawyer, 1990; Monroe \& Lee, 1999). As a case in point, Proctor and Gamble’s Tide detergent owns a commanding 44 percent market share in the powder detergent category, four times larger than the share of the closest competitor, the firm's own Gain brand (Walsh \& Hume, 2000). Yet in Study 2, consumers consistently rated a masked version of Tide as the least attractive alternative - except when they were exposed to both number-of-uses-per-package and price-per-use cues.

This finding suggests interesting explanations and questions for future research. First, it points to the power brand promotions, consumer loyalty to certain brands, and perhaps even the influence of shelf-facing dominance on consumer choice. It also reinforces the research results reported by Hoyer (1984) and Dickson and Sawyer (1990) that consumers often spend little time at the point of purchase evaluating products such as laundry detergent. If consumers were more diligent in evaluating the per-wash value of Tide compared to other brands, the market share discrepancy between Tide and its competitors might be less pronounced. Another explanation that is consistent with the present research is that consumers may find it difficult (or not worth the effort) to derive an accurate assessment of value and then develop a brand-preference ranking using the pricing information currently available on supermarket shelves.

The discrepancy between Tide’s actual market share and our findings highlights a limitation of Study 2. First, in order to control for the confounding influence of brand name, it was necessary to use fictitious brands, which obviously removed an important attribute consumers consider when choosing laundry detergents. Second, the use of artificial (paper and pencil) stimuli only approximated and probably simplified the decision environment consumers actually face. Third, subjects in both studies were free to use as much time as they wished to make their evaluations and may have devoted greater cognitive resources to the task than would typically occur during an actual supermarket visit. Similarly, because subjects were asked to judge brands as opposed to make a choice (see Johnson \& Russo, 1984), they may have paid attention to price cues that would typically be ignored or minimized, and used a more systematic information-integration strategy than would commonly be used when making grocery purchases.

One reviewer suggested an interesting limitation to usage-based measures. For a usagebased comparison of items in a product category to be fully accurate and thus most useful, the consumer must use the product in accordance with the usage-based measure. For laundry detergents, the consumer must use the correct quantity of detergent per wash load. For breakfast cereals, the consumer must eat substantially the correct portions. As the reviewer speculated, this may be a particularly important point when there are multiple members of the household who might use the product and use it differently, even to the point where the purchaser understands portion control but also may expect other members of the household will over use the product. 
This suggests the need for both survey-based and observational research to get an understanding of how well usage measures are being used. While it is likely to be the case that usage-based measures are valuable, they, like the measure-based unit price, may not be as useful as they could be if they were used more effectively.

\section{CONCLUSION}

The results of this study suggest that in many product categories, measure-based unit pricing does not always provide the right information for consumers to make an accurate evaluation of brands' true value. Although a usage-based pricing scheme suffers from the challenge of setting usage standards, consumers are more likely to use this index than current measure-based measures, particularly when they are confronted with products of different strengths and usage rates displayed side-by-side on retailer shelves. A usage-based unit price index would also serve to facilitate the launch of new products with different formulas. If consumers learn to judge concentrated products based on usage rates rather than a complicated evaluation of total-price, measure-based unit-price, and package-volume cues, it is reasonable to expect that they will make better purchase decisions, and a more smooth transition to concentrated products in many different categories would occur.

\section{REFERENCES}

Aaker, D., \& Ford, G. T. (1983). Unit Pricing Ten Years Later: A Replication, Journal of Marketing, 47, 118-122.

Appelbaum, C. (1991), “Colgate Readies New Ads for Fresh Start,” Adweek, March 11, 10.

Bauer, B. (1994). Dynamic Detergents; The Popularity of Ultra Concentrates is Forcing Most

Retailers to Frequently Reset the Laundry Aisle. Supermarket News, 44 (15), April 11, 39.

Bettman, J.R., Johnson, E.J., \& Payne, J.W. (1991). Consumer Decision Making,” in Handbook of Consumer Behavior, Robertson, T.S \& Kassarjian, H.H., eds., Englewood Cliffs, NJ: Prentice Hall.

Breskin, I. (1995). Consumers Resist Increased Liquid Concentration, Chemical Week, 156 (3), January 25, 39.

Cohen, J., \& Cohen, P. (1983). Applied Multiple Regression/Correlation Analysis for the Behavioral Sciences, $2^{\text {nd }}$ edition, Hillsdale NJ: Lawrence Erlbaum Associates.

Consumers Union of the U.S., Inc. (1995), Laundry Detergents. Do Good Things Come in Small Packages?” Consumer Reports, 60 (February), 92.

“Consumer-friendly Labels Sought for Liquid Laundry Soap” (1995), 29, Marketing News, January 30, 9.

Creyer, E.H., \& Ross, W.T. (1997). Tradeoffs between Price and Quality: How a Value Index Affects Preference Formation, Journal of Consumer Affairs, 31, 280-302.

Dickson, P.R., \& Sawyer, A.G. (1990). The Price Knowledge and Search of Supermarket Shoppers, Journal of Marketing, 54, 42-53.

Garman, E. Thomas (1996), Consumer Economic Issues in America, 4th edition, Houston, TX: Dame Publications.

Granger, C.W., \& Billson, A. (1972). Consumers’ Attitudes Toward Package Size and Price, Journal of Marketing Research, 239-248. 
Hoyer, W. D. (1984). An Examination of Consumer Decision Making for a Common Repeat Purchase Product, Journal of Consumer Research, 822-829.

Isakson, H.R., \& Maurizi, A.R. (1973). The Consumer Economics of Unit Pricing, Journal of Marketing Research, 10, 277-285.

Johnson, E.J., \& Russo, J.E. (1984). Product Familiarity and Learning New Information, Journal of Consumer Research, 11, 542-550.

Johnson, E.J., \& Payne, J.W. (1985). Effort and Accuracy in Choice, Management Science, 31, 395-414.

Johnson, E. J., Payne, J.W., \& Bettman, J.R. (1988). Information Displays and Preference Reversals, Organizational Behavior and Human Decision Processes, 42, 1-21.

Manning, KC., Sprott, D.E., \& Miyazaki, A.D. (1998). “Consumer Responses to Quantity Surcharges: Implications for Retail Price Setters,” Journal of Retailing, 74 (Fall), 373-99.

McElroy, B.F., \& Aaker, D.A. (1979). Unit Pricing Six Years After Introduction, Journal of Retailing, 3, 44-57.

Mitchell, V.-W., Lennard, D., \& McGoldrick, P. (2003). “Consumer Awareness, Understanding and Usage of Unit Pricing,” British Journal of Management, 14, 173-187.

Miyazaki, A.D., Sprott, D.E., \& Manning, K. (2000). Unit Prices on Retail Shelf Labels: An Assessment of Information Prominence, Journal of Retailing, 76, 93-112.

Monroe, K.B., \& Lee, A.Y. (1999). Remembering Versus Knowing: Issues in Buyers’ Processing of Price Information, Journal of the Academy of Marketing Science, 27, 207-225.

Mullin, R. (1992), “Canada Was Lukewarm on Concentrates in '91,” Chemical Week, January 29, 42 and 44.

Neff, J. (1999), “Clorox Joins Bandwagon for Ultra-Strength Brands,” Advertising Age, November 29, 14.

Russo, J.E. (1977). The Value of Unit Price Information, Journal of Marketing Research, 14, 193-201.

Russo, J.E., Kreiser, G., \& Miyashita, S. (1975). An Effective Display of Unit Price Information, Journal of Marketing, 39, 11-19.

Slovic, P. (1972). From Shakespeare to Simon: Speculations - and Some Evidence About Mom’s Ability to Process Information, Oregon Research Institute Monograph, 12 (2), Eugene, OR.

Turcsik, R. (1994). A Powerful Formula: Liquid Detergents, Led by Ultra Concentrates, are Showing Strength in a Lackluster Category, Supermarket News, 44 (November 7), 37-38,42.

Walsh, T., \& Hume, C. (1999). Soaps and Detergents - Sharing the Risks and Rewards, Chemical Week, 161 (January 27), 25-28.

Walsh, T., \& Hume, C. (2000). Soaps and Detergents - Rising Costs Burst Soapers’ Bubbles, Chemical Week, 162 (January 26), 27-29.

Zeithaml, V.A. (1982). Consumer Response to In-Store Price Environments, Journal of Consumer Research, 8, 357-369. 
Table 1

Stimuli for Study 1

Laundry Detergent

\begin{tabular}{|c|c|c|c|c|c|}
\hline & Brand A & Brand B & Brand C & Brand D & Brand E \\
\hline Weight & $58 \mathrm{oz}$. & $100 \mathrm{oz}$. & $85 \mathrm{oz}$. & $78 \mathrm{oz}$. & $87 \mathrm{oz}$. \\
\hline Price & $\$ 4.79$ & $\$ 6.03$ & $\$ 5.59$ & $\$ 5.59$ & $\$ 7.27$ \\
\hline Unit Price & $\$ .83 / \mathrm{oz}$. & $\$ .60 / \mathrm{oz}$. & $\$ .66 / \mathrm{oz}$. & $\$ .72 / \mathrm{oz}$. & $\$ .84 / \mathrm{oz}$. \\
\hline Price per Use & $\$ .21$ & $\$ .19$ & $\$ .17$ & $\$ .14$ & $\$ .18$ \\
\hline Uses/Package & 22 & 31 & 33 & 40 & 40 \\
\hline
\end{tabular}

Note: Brand B is lowest cost in terms of price per ounce; Brand D is lowest cost in terms of price per usage.

\section{Breakfast Cereal}

\begin{tabular}{|c|c|c|c|c|c|}
\hline & Brand A & Brand B & Brand C & Brand D & Brand E \\
\hline Weight & $18 \mathrm{oz}$. & $16 \mathrm{oz}$. & $17 \mathrm{oz}$. & $16 \mathrm{oz}$. & $15 \mathrm{oz}$. \\
\hline Price & $\$ 3.19$ & $\$ 3.13$ & $\$ 3.11$ & $\$ 2.69$ & $\$ 2.83$ \\
\hline Unit Price & $\$ .17 / \mathrm{oz}$. & $\$ .19 / \mathrm{oz}$. & $\$ .18 / \mathrm{oz}$. & $\$ .16 / \mathrm{oz}$. & $\$ .18 / \mathrm{oz}$. \\
\hline Price per Use & $\$ .35$ & $\$ .39$ & $\$ .28$ & $\$ .33$ & $\$ .35$ \\
\hline Uses/Package & 9 & 8 & 11 & 8 & 8 \\
\hline
\end{tabular}

Note: Brand D is lowest cost in terms of price per ounce; Brand C is lowest cost in terms of price per usage. 
Table 2

Results for Study 1: Coefficients (Statistical Significance)

\begin{tabular}{|c|c|c|c|c|}
\hline \multirow[b]{2}{*}{ Independent Variable } & \multicolumn{2}{|c|}{ Laundry Detergent } & \multicolumn{2}{|c|}{ Breakfast Cereal } \\
\hline & $\mathbf{B}$ & D & D & C \\
\hline & $1^{\text {st }}$ price/oz & $\mathbf{1}^{\text {st }}$ price/use & $1^{\text {st }}$ price/oz & $1^{\text {st }}$ price/use \\
\hline Price per Use & $-0.929(.022)$ & NS & NS & NS \\
\hline Price per Ounce & $+0.675(.089)$ & $-0.637(.032)$ & $+0.726(.038)$ & $-0.849(.005)$ \\
\hline Description Provided & NS & NS & NS & $+0.542(.071)$ \\
\hline Ranking Elicited & NS & NS & $-0.589(.085)$ & NS \\
\hline Model Chi $^{2}$ (4 df) & 11.813(.019) & 8.252(.083) & $15.626(.004)$ & $8.909(.063)$ \\
\hline
\end{tabular}


Table 3

Product profile stimuli for Study 2

\begin{tabular}{|c|c|c|c|c|c|c|c|}
\hline Brand & Concentration & Weight & Total Price & Wash load & $\begin{array}{l}\text { Unit } \\
\text { Price } \\
\end{array}$ & $\begin{array}{c}\text { Price Per } \\
\text { Wash }\end{array}$ & $\begin{array}{l}\text { Correct } \\
\text { Ranking }\end{array}$ \\
\hline $\begin{array}{l}\text { Brand } 1 \\
\text { (Tide) }\end{array}$ & $\begin{array}{l}\text { 1/4 cup ultra: } \\
2.2 \mathrm{oz}\end{array}$ & $\begin{array}{l}\text { Set 1: } 92 \mathrm{oz} . \\
\text { Set 2: } 39 \mathrm{oz} \text {. } \\
\text { Set 3: } 92 \mathrm{oz} .\end{array}$ & $\begin{array}{l}\text { Set 1: } \$ 9.39 \\
\text { Set 2: } \$ 4.59 \\
\text { Set 3: } \$ 9.39\end{array}$ & $\begin{array}{l}\text { Set 1: } 42 \\
\text { Set 2: } 18 \\
\text { Set 3: } 42\end{array}$ & $\begin{array}{c}\$ 1.63 / \mathrm{lb} \\
.\end{array}$ & $\$ 0.22$ & 3 \\
\hline $\begin{array}{c}\text { Brand } 2 \\
\text { (Arm \& } \\
\text { Hammer) }\end{array}$ & $\begin{array}{c}\text { 4/10 cup ultra: } \\
3.2 \text { oz. }\end{array}$ & $\begin{array}{l}\text { Set 1: } 96 \mathrm{oz} . \\
\text { Set 2: } 96 \mathrm{oz} . \\
\text { Set 3: } 135 \mathrm{oz} \text {. }\end{array}$ & $\begin{array}{l}\text { Set 1: } \$ 4.49 \\
\text { Set 2: } \$ 4.49 \\
\text { Set 3: } \$ 6.25\end{array}$ & $\begin{array}{l}\text { Set 1: } 30 \\
\text { Set 2: } 30 \\
\text { Set 3: } 42\end{array}$ & $\$ 0.75 / \mathrm{lb}$ & $\$ 0.15$ & 1 \\
\hline $\begin{array}{l}\text { Brand } 3 \\
\text { (Purex) }\end{array}$ & $\begin{array}{l}1 \text { cup regular } \\
\text { formula: } 8 \mathrm{oz} \text {. }\end{array}$ & $\begin{array}{l}\text { Set 1: } 104 \mathrm{oz} . \\
\text { Set 2: } 104 \mathrm{oz} . \\
\text { Set 3: } 335 \mathrm{oz} .\end{array}$ & $\begin{array}{l}\text { Set 1: } \$ 4.29 \\
\text { Set 2: } \$ 4.29 \\
\text { Set 3: } \$ 12.99\end{array}$ & $\begin{array}{l}\text { Set 1: } 13 \\
\text { Set 2: } 13 \\
\text { Set 3: } 42\end{array}$ & $\$ 0.66 / \mathrm{lb}$ & $\$ 0.33$ & 4 \\
\hline $\begin{array}{l}\text { Brand } 4 \\
\text { (Bold) }\end{array}$ & $\begin{array}{l}\text { 1/4 cup ultra: } \\
2 \mathrm{oz} .\end{array}$ & $\begin{array}{l}\text { Set 1: } 85 \mathrm{oz} . \\
\text { Set 2: } 48 \mathrm{oz} \text {. } \\
\text { Set 3: } 85 \mathrm{oz} .\end{array}$ & $\begin{array}{l}\text { Set 1: } \$ 6.99 \\
\text { Set 2: } \$ 4.56 \\
\text { Set 3: } \$ 6.99\end{array}$ & $\begin{array}{l}\text { Set 1: } 42 \\
\text { Set 2: } 24 \\
\text { Set 3: } 42\end{array}$ & $\begin{array}{c}\$ 1.32 / \mathrm{lb} \\
.\end{array}$ & $\$ 0.17$ & 2 \\
\hline
\end{tabular}


Table 4

Value Assessment ANOVA for Study 2

Source df $\begin{array}{r}\text { Sum of } \\ \text { Squares }\end{array} \quad$ F-Statistic P-Value

BETWEEN SUBJECTS

Measure-based Unit Price

Wash load

Usage-based Unit Price

Wash load by Measure-based Unit Price

Usage-based Unit Price by Measure-based Unit Price

Usage-based Unit Price by Wash load

Usage-based Unit Price by Wash load by Measurebased Unit Price

Between Subjects Error

\section{WITHIN SUBJECTS}

Choice Set

Choice Set by Measure-based Unit Price

Choice Set by Wash load

Choice Set by Usage-based Unit Price

Choice Set by Measure-based Unit Price by Wash load

Choice Set by Measure-based Unit Price by Usage-based Unit Price

Choice Set by Wash load by Usage-based Unit Price

Choice Set by Measure-based Unit Price by Usage-based Unit Price by Wash load

Within Subjects Error Squares

$\begin{array}{cccc}1 & 0.487 & 0.530 & \text { NS } \\ 1 & 16.944 & 18.443 & .000 \\ 1 & 56.459 & 61.457 & .000 \\ 1 & 0.083 & 0.090 & \text { NS } \\ 1 & 0.623 & 0.678 & \text { NS } \\ 1 & 12.364 & 13.458 & .000 \\ 1 & 2.753 & 2.997 & .084\end{array}$

$338 \quad 310.513$

$2 \quad 21.411 \quad 23.117 \quad .000$

2

0.955

1.031

NS

$2 \quad 1.162 \quad 1.254 \quad$ NS

$\begin{array}{llll}2 & 0.597 & 0.644 & \text { NS }\end{array}$

$\begin{array}{llll}2 & 0.943 & 1.018 & \text { NS }\end{array}$

$\begin{array}{llll}2 & 0.290 & 0.313 & \text { NS }\end{array}$

$\begin{array}{llll}2 & 0.115 & 0.124 & \text { NS }\end{array}$

$\begin{array}{llll}2 & 1.622 & 1.751 & \text { NS }\end{array}$

$676 \quad 313.04$ 
Table 5

Average z-scores for Statistically Significant Effects for Table 2 (95\%Confidence Interval Lower and Upper Bounds)

\section{MAIN EFFECTS}

\begin{tabular}{|c|c|c|c|c|}
\hline & Absence & Presence & & \\
\hline Measure-based Unit Price & $\begin{array}{c}.995 \\
(.852-1.138)\end{array}$ & $\begin{array}{c}.920 \\
(.775-1.064)\end{array}$ & & \\
\hline Wash load & $\begin{array}{c}.735 \\
(.593-.877)\end{array}$ & $\begin{array}{c}1.180 \\
(1.034-1.326)\end{array}$ & & \\
\hline \multirow[t]{2}{*}{ Usage-based Unit Price } & $\begin{array}{c}.551 \\
(.413-.690)\end{array}$ & $\begin{array}{c}1.363 \\
(1.214-1.513)\end{array}$ & & \\
\hline & Same Size & Same Price & Same Wash loads & \\
\hline Choice Set & $\begin{array}{c}.766 \\
(.659-.873)\end{array}$ & $\begin{array}{c}.991 \\
(.871-1.112)\end{array}$ & $\begin{array}{c}1.115 \\
(.990-1.239)\end{array}$ & \\
\hline \multirow[t]{2}{*}{$\begin{array}{l}\text { SIGNIFICANT } \\
\text { INTERACTION }\end{array}$} & & & & \\
\hline & Neither & $\begin{array}{l}\text { Wash load } \\
\text { Only }\end{array}$ & Usage-based Only & Both \\
\hline $\begin{array}{l}\text { Usage-based Unit Price by } \\
\text { Wash load }\end{array}$ & $\begin{array}{c}-.139 \\
(-.495-.328)\end{array}$ & $\begin{array}{c}.964 \\
(.761-1.166)\end{array}$ & $\begin{array}{c}1.331 \\
(.119-1.543)\end{array}$ & $\begin{array}{c}1.396 \\
(.185- \\
1.607)\end{array}$ \\
\hline
\end{tabular}

